\section{GRP-012 ADHERENCE, PERSISTENCE AND FINANCIAL EVALUATION IN THE TREATMENT OF PROSTATE CANCER}

doi:10.1136/ejhpharm-2013-000276.012

P Sorice, F Santoleri, R Lasala, RC Rizzo, A Costantini. General Hospital of Pescara, Hospital Pharmacy, Pescara, Italy

Background The success of home treatment is strongly influenced by patient adherence to treatment. Non-adherence to treatment represents not only an important issue for the patient, affecting both the clinical efficacy and safety of the drug treatment, but also has financial and social implications for the community.

Purpose This study evaluated the adherence to treatment, persistence, and the daily cost of treatment in patients with prostate cancer treated with gonadotropin-releasing hormone agonists, comparing leuprorelin 3.75-11.25, leuprorelin 7.5-22.5 and triptorelin.

Materials and Methods Adherence to treatment was measured as the ratio between the Received Daily Dose (RDD) and the Prescribed Daily Dose (PDD), using software developed for this purpose by hospital pharmacists. The RDD was calculated as the sum of the number of days between two consecutive drug refills, whilst the PDD was determined based on the treatment regimen as prescribed by the physician. The persistence was calculated as the sum of the number of days the patient had stayed on treatment.

The cost of daily treatment was calculated on the basis of the RDD.

Results 126 patients were enrolled in this study for triptorelin, 143 for leuprorelin 3.75-11.25 and 31 for leuprorelin 7.5-22.5. The adherence values for all drugs ranged between 0.95 and 1.10, showing good quality management of domiciled treatment. The analysis of persistence conducted over three years showed a decrease by $20 \%$ for leuprorelin $3.75-11.25,25 \%$ for triptorelin and $50 \%$ for leuprorelin 7.5-22.5. The cost per RDD was $€ .2 .15, € .2 .24$ and $€ .2 .84$ for leuprorelin 7.5-22.5, leuprorelin 3.75-11.25 and triptorelin respectively.

Conclusions The excellent adherence values showed that all the drugs studied have a good safety profile and easy administration. In fact, patients complied with the dosage and medication regimens as recommended by prescribers. The persistence values were overlapping. The cost per RDD for triptorelin was $23 \%$ higher than leuprorelin.

No conflict of interest.

\section{GRP-013 ADVERSE DRUG REACTIONS IN THALIDOMIDE TREATED PATIENTS}

doi:10.1136/ejhpharm-2013-000276.013

T Gramage Caro, S Orbe Izquierdo, C Pérez Menéndez-Conde, C Palomar Fernández, M Muñoz García, T Bermejo Vicedo. Hospital Ramón y Cajal, Pharmacy, Madrid, Spain

Background Thalidomide is a chemotherapeutic agent approved by the EMA for multiple myeloma treatment. It is considered a high risk drug and should be prescribed and dispensed within a special pharmacovigilance programme.

Purpose To evaluate the incidence of adverse drug reactions (ADRs) to thalidomide; to analyse their type and severity.

Materials and Methods Retrospective cohort study, conducted between January 2008 and December 2011 in a university hospital. Patients treated with thalidomide were selected through the Pharmacy Department Outpatient Unit medicines records.

Patient clinical records were reviewed. Any doubts were checked with the attending physicians.

Data recorded: personal data (age, gender), main diagnosis, thalidomide ADRs, start and end dates of both thalidomide treatment and ADRs.
ADR incidence was calculated. Association between ADRs and thalidomide discontinuation was determined.

ADR causal relation was determined by the Karch Lasagna algorithm (definite, probable, possible, conditional). ADR type was classified according to the Rawlins and Thompson classification (type A: dose-dependent or type B: not dose-dependent) and ADR severity and outcome according to Spanish Pharmacovigilance System criteria.

Results Twelve patients were included (mean age $59 \pm 12$ years, $50 \%$ men).

Medical diagnosis: multiple myeloma 91.66\% (11 patients) and cutaneous, vascular and digestive systemic sclerosis $8.3 \%$ (1).

The incidence of thalidomide ADRs was $83.3 \% .8 .3 \%$ (1) of treatment discontinuations were due to thalidomide ADR.

$64.71 \%$ (11) of patients showed neurotoxicity, 17.64\% (3) blood disorders, $11.76 \%$ (2) oedema and $5.88 \%$ of them (1) digestive disorders

ADRs detected were type A (dose-dependent) in $100 \%$ of cases (17 patients), probable in $41.18 \%$ (7), and possible in $58.82 \%$ (10) of them.

Overall, $41.18 \%$ of ADRs were severe (7). ADR outcomes: $64.70 \%$ of ADRs (11 patients) were resolved, $17.65 \%$ (3) unresolved and $17.65 \%$ (3) were classified as 'death unrelated to the drug'.

Every ADR detected was notified to the Spanish Pharmacovigilance System.

Conclusions Although the incidence of thalidomide ADRs was high (83.3\%), ADRs only caused treatment discontinuation in $8.3 \%$ of cases.

Neurotoxicity was the most frequent ADR.

Almost half of patients had severe ADRs and these did not resolve in $17.65 \%$ of cases.

No conflict of interest.

\section{GRP-014 AN E-LEARNING PROGRAMME ON HIGH-RISK DRUGS - DOES IT ACTUALLY INCREASE USER KNOWLEDGE?}

doi:10.1136/ejhpharm-2013-000276.014

${ }^{1} \mathrm{M}$ Creed, ${ }^{1} \mathrm{M}$ McGuirk, ${ }^{1} \mathrm{C}$ Meegan, ${ }^{2 \mathrm{D}}$ Murray. ${ }^{1}$ Mater Misericordiae University Hospital, Pharmacy Department, Dublin, Ireland (Rep.); ${ }^{2}$ Peamount Hospital, Pharmacy Department, Dublin, Ireland (Rep.)

Background High-risk drugs are involved in serious medicines errors. Studies have identified a range of contributory factors including lack of training. The MMU Hospital developed a E-learning programme 'A Guide to High-risk Drugs' to enable teaching; incorporating an inbuilt evaluation tool to assess the learning outcome.

Purpose To evaluate the learning from undertaking an e-learning programme on high-risk drugs.

To ascertain if the programme is suitable for different types of institutions.

To identify user knowledge deficits.

Materials and Methods The programme was trialled in two different hospitals. The MMUH, a 600 bed acute hospital and Peamount Hospital, a 380-bed rehabilitation and continuing care hospital. The participants were qualified Doctors, Nurses and Pharmacists. All 170 participants undertook 20 pre-assessment questions followed by the programme then the same questions in a post-assessment. Results from each institution and discipline were analysed.

Results 29 Interns completed the programme at the MMUH and 11 SHOs/Registrars in Peamount. A mean pre-assessment score of $58 \%$ (MMUH) and $56 \%$ (Peamount) increased to a post score of $83 \%$ in both hospitals. MMUH Nurses $(n=38)$ yielded an improvement, $48 \%$ to $73 \%$; and Peamount Nurses ( $n=40$ ), $39 \%$ to $65 \%$. MMUH Pharmacists $(\mathrm{n}=20$ ) improved from $83 \%$ to $94 \%$. 
Individual questions were further analysed to ascertain if there were particular drugs causing difficulty. Analysis showed that a question on potassium chloride yielded low pre-assessment scores of $21 \%$ and $39 \%$ respectively for MMUH Doctors and Nurses and $45 \%$ and $20 \%$ for Peamount. Although both disciplines improved, this demonstrated a need for further training with this drug.

Conclusions The e-learning programme showed a significant increase in user knowledge, in both hospitals, for all disciplines. These results are very encouraging given the differences between the institutions, grades of staff and experience. The results do not stem from a 'specific teacher effect' and therefore are reproducible in multiple sites.

No conflict of interest.

\section{GRP-015 AN OVERVIEW OF HOSPITAL PHARMACEUTICAL EXPENDITURE IN GREECE OVER THE LAST TWO YEARS}

doi:10.1136/ejhpharm-2013-000276.015

'K Nikou, ${ }^{2} \mathrm{~K}$ Perdikouri, ${ }^{3} \mathrm{M}$ Katharaki. 'General Hospital of Chest Diseases "SOTIRIA", Pharmacy, Athens, Greece; 'Spiliopoulio Hospital, Pharmacy, Athens, Greece; ${ }^{3} G e n e r a l$ Hospital "Alexandra", Nursing Services Division, Athens, Greece

Background Under the burden of the economic crisis in Greece, a series of cost containment measures for hospitals' operating costs have been implemented, with the emphasis put on limiting the money spent on medicines purchasing.

Purpose To review the extent to which the target of reducing hospital pharmaceutical expenses has been achieved along with reporting the changes, as far as the 'in hospital' use of generic medicine is concerned.

Materials and Methods Financial data from 136 Greek public hospitals, as officially reported in ESY.net database, were collected and compared for the years of interest. The financial data were selected with respect to the cost of purchasing medicines and nonpharmaceutical material, while other operational costs were omitted. Results The cost of purchasing medicines constantly accounts for a high percentage of a hospital's budget for supplies $(51 \%, 53 \%$ and $56 \%$ for 2010, 2011 and 2012 respectively). An overall decrease in pharmaceutical expenditures was achieved (23\% reduction in 2011, along with a further reduction of $17 \%$ in 2012). Although rates of introducing generic drugs differ among different hospitals, an increase in use of generics was observed (26\% in 2011 and 30\% in 2012). Psychiatric hospitals seem to have better scores compared to paediatric and oncology departments.

Conclusions The 'in hospital' use of generic drugs score is significantly higher compared to that of the Greek market in general $(18 \%)$ and has therefore contributed to the hospitals' attempt to reduce the amount of money spent on medicine supplies. The lower rates of generics' use observed in paediatric hospitals are consistent with the lower possibility for substitution in these cases. Last but not least, when selecting and implementing drug cost management strategies, it is essential that pharmacists remain mindful of patient safety and quality of patient care.

No conflict of interest.

\section{GRP-016 ANALYSIS AND CONSUMPTION OF INNOVATIVE ANTIDIABETIC DRUGS IN PIEDMONT PATIENTS}

\section{doi:10.1136/ejhpharm-2013-000276.016}

V Colombardo, V Besso, R Bona, M Conson, M Nocera, M Sampietro, I Barbato, P Mero, D Piccioni, S Martinetti. Hospital Pharmacy, ASL AT, Asti, Italy

Background The increase in deaths due to diabetes records a trend in growth and the OSMED National Report of 2011 highlights a prescription shift towards the high-cost innovative drugs for the treatment of type II diabetes mellitus (DMII). This is subject to intensive monitoring by the health ministry.

In the management of diabetic patients, the guidelines suggest an early intensive therapeutic intervention and the pursuit of a personal glycaemic target for avoiding hypoglycaemic episodes, which are possibly responsible for the increased risk of developing cardiovascular episodes.

Purpose To analyse the population, consumption and type of innovative diabetic drugs used in the Piedmont region; this is to put a value on the type of treatment used for DMII, because the advantages of innovative therapy must be valued too.

Materials and Methods In the first step the incidence of DMII in Piedmont was valued by analysing data from the regional diabetic database during the period 2007-2012. Dipeptidyl peptidase 4 inhibitors (sitagliptin and vildagliptin alone or in association with metformin and saxagliptin), thiazolidinediones (pioglitazone alone or in association with metformin), glucagon-like peptide 1 (exenatide, liraglutide), insulin glargine and detemir were considered innovative drugs. Consumption and type of drugs were analysed in terms of the defined daily dose/1000 inhabitants/day (DDD) over a six-month period in 2012 using regional databases of prescriptions which enabled us to access population data. 2012 data were compared with 2011.

Results The first striking finding is the increase in the incidence of DMII, $1.70 \%$ in five years, which corresponds to 80,327 patients. Focusing on the population treated with innovative drugs revealed that $21 \%(61,679 / 294,590)$ of diabetic patients are 65 years old and far more males than females are affected (respectively $53.87 \%$ vs. $46.13 \%$ ). The drug most used is insulin glargine with $43.84 \%$ of total consumption, another $25.08 \%$ use DPP4 inhibitors alone or in association, $20.05 \%$ use pioglitazone alone or in association, $9.02 \%$ use glucagon-like peptide 1 and $2.01 \%$ use insulin detemir. The comparison with the same period of 2011 highlights the increased consumption of innovative drugs in Piedmont, 23\% (8.97 DDD in 2012 vs. 6.91 DDD in 2011) while Italian data record an increase of $5 \%$ (15.69 DDD vs. 14.87 in 2011).

Conclusions Increased consumption of these drugs suggests that medical prescriptions could maybe move on innovative therapeutic molecules. It is important that clinicians discuss and compare the data analysis shown above with medical management guidelines, with the aim of estimating the genuine advantages of innovative drugs in terms of compliance, reduction in adverse reactions and increased quality of life.

No conflict of interest.

\section{GRP-017 ANALYSIS AND PREVENTION OF MUSCULOSKELETAL DISORDERS IN A HOSPITAL STERILISATION UNIT}

doi:10.1136/ejhpharm-2013-000276.017

L Tortolano, I Becamel, D Briand, F Vincent. Hopital Européen Georges Pompidou, Paris, France

Background Musculoskeletal disorders (MSDs) are problems caused by the poor ergonomic design of work stations. The daily work of sterilisation, especially carrying heavy loads, carries a risk of developing MSDs.

Our sterilisation unit, certified ISO 9001, tries to improve the working environment including the ergonomics of work stations.

Purpose To establish an inventory of fixtures and to suggest preventive measures in order to limit the appearance of those disorders.

Materials and Methods The whole of the sterilisation unit workforce was interviewed about any pain, physical effort and nonergonomic situations that they routinely face during their daily work. 